\title{
Effect of Curcuma longa tuber powder extract on size of silver nanoparticles prepared by green method
}

\begin{abstract}
Biosynthesis of noble metal nanoparticles is a vast developing area of research. In the present study, silver nanoparticles (Ag-NPs) were synthesized from aqueous silver nitrate through a simple and biosynthetic route using water extract of Curcuma longa (C. longa) tuber powder, which acted simultaneousl as a reductant and stabilizery. The as-prepared samples are characterized using UV-Visible, XRD, TEM, SEM, EDXF, and FT-IR techniques. The formation of Ag-NPs is evidenced by the appearance of the signatory brown color of the solution and $\mathrm{UV}$-vis spectra. Formation of $\mathrm{Ag} / \mathrm{C}$. longa was determined by $\mathrm{UV}-\mathrm{Vis}$ spectroscopy where surface plasmon absorption maxima can be observed at $457-415 \mathrm{~nm}$ from the UV-Vis spectrum. The XRD analysis shows that the Ag-NPs are of a face-centered cubic structure. Well-dispersed Ag-NPs with anisotropic and isotropic morphology for 5, 10, and $20 \mathrm{~mL}$ of $\mathrm{C}$. longa water extract having a size less than $10 \mathrm{~nm}$ are seen in TEM images. The optimum volume extraction to synthesize smallest particle size was $20 \mathrm{~mL}$ with mean diameter and standard division $4.90 \pm 1.42 \mathrm{~nm}$. FT-IR spectrum indicates the presence of different functional groups in capping the nanoparticles with $\mathrm{C}$. longa. The zeta potential analysis results indicated that the charge of $\mathrm{C}$. longa was negative and increased in $\mathrm{Ag} / \mathrm{C}$. longa emulsion with increasing of volumes of extract used $(10-20 \mathrm{~mL})$. The most needed outcome of this work will be the development of value-added products from $\mathrm{C}$. longa for biomedical and nanotechnology-based industries.
\end{abstract}

Keyword: Silver nanoparticles; Curcuma longa; Biosynthesis; Green synthesis; Zeta potential analysis 